has not proved very successful, but Knouf and others report I2 cases with 9 recoveries, 5 of whom were under two years of age. They were all treated with a continuous drip of sodium sulphapyridine, 0.5 or I per cent in normal saline. Sako and others also report 7 cases under four years old with 5 recoveries. These cases were treated with large doses of sulphadiazine orally. A total of 80 to $90 \mathrm{gm}$. of the drug was given to children aged 2 to 3 years, and the drug was given in reduced doses after apparent cure for at least two weeks. The comment of Dr. Stanley Banks in the Medical Annual is worth quoting. "These papers provide a valuable pointer to the intensity and duration of the sulphonamide treatment necessary for success in this dire disease. Treatment on the ordinary lines appropriate for meningococcal meningitis has shown many failures."

\title{
REFERENCES
}

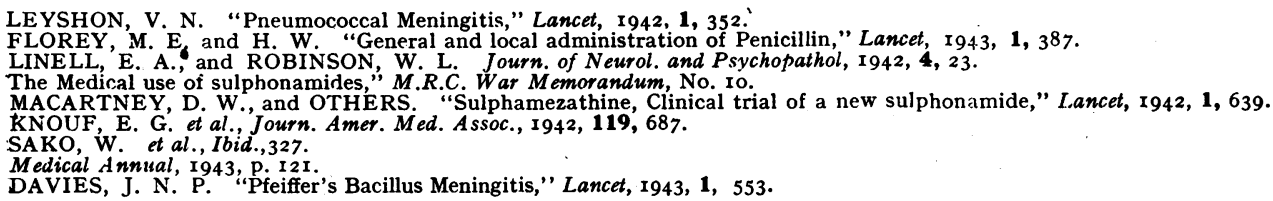

ENCEPHALITIS

\author{
By LEWIS R. YEALLAND, M.D., F.R.C.P. \\ (Phys. Prince of Wales Hosp. and West End Hosp. for Nervous Diseases, etc.)
}

Inflammation of the brain may occur as a primary disease, but it is most frequently due to an extension of infection from other parts. A combined inflammation of the brain and spinal cord may occur, giving rise to a condition which is called encephalo-myelitis. The lesion of poliomyelitis is not always limited to the spinal cord, it sometimes extends into the brain stem or farther up into the brain. This is called polioencephalitis superior. Encephalitis may result from an extension of disease, (a) from the meninges in tuberculous, epidemic or other form of meningitis, meningo-encephalitis; (b) from the aural or nasal sinuses, or from bronchiectasis, suppurative encephalitis (abscess of the brain); and from (c) injuries of the skull, traumatic encephalitis. Further, encephalitis may occur as a complication of the specific fevers, and, in particular measles and scarlet fever; and vaccination. Finally, disseminated patches of encephalitis are to be found sometimes in syphilis when there is no evidence of that disease elsewhere.

Particular attention will be directed here to the following four types of non-suppurative encephalitis, all of which may be regarded as rare: (i) Encephalitis lethargica. (ii) Encephalitis periaxialis. (iii) Acute disseminated encephalomyelitis. (iv) Otogenic encephalitis. The first three of these are primary; the last named is secondary to infection in the ear.

\section{DIAGNOSIS OF ENCEPHALITIS}

In the early phases of encephalitis there may be general symptoms of pyrexia, headache, irritability, convulsions, delirium and vomiting. On the other hand, there may be coma more or less profound. Neurological signs do not, as a rule, make their appearance during the first day or two of the disease, and the condition is often called influenza if no focus of infection can be discovered. Indeed the acute infectious initial phase tends to mask the neurological features. Moreover, when intracranial localising signs are brought to light, the acute infectious phase may have passed off and such indications of intracranial disease as, for example, hemiplegia or aphasia may suggest other causes than that of encephalitis. In these circumstances difficulties in diagnosis arise, not as to where the lesion is situated, but as to what is its 
nature, inflammatory or vascular. Kinnier Wilson when teaching often referred to the "EATIT tip," whose origin he attributed to Hughlings Jackson, as a means of help in distinguishing the nature of all neurological lesions. It is based on the time element, that is, the time that elapses between the occurrence of the first symptoms of the illness and the manifestation of neurological signs, embolism being the quickest and tumour the slowest. This method of diagnosis is helpful, but must not be regarded as accurate.

Embolus
Apoplexy (haemorrhage).
Thrombosis
Inflammation
Tumour

seconds

minutes

hours

days

weeks or months

\section{- ENCEPHALITIS LETHARGICA}

In I9I6, when I was resident medical officer at the National Hospital, Queen Square, there was admitted, under the care of Dr. James Taylor, a woman who presented partial bilateral external ophthalmoplegia, ptosis and profound drowsiness with a history of influenza during the past fortnight. About the same period, Dr. F. Batten, who was physician to that hospital as well as to the neighbouring sick children's hospital, showed me two cases there which had been recently admitted with similar clinical features, but whose chief interest was to be found in the fact that they exhibited a marked degree of cerea flexibilititas. Dr. Batten was of the opinion that all these cases were manifestation of the same disease. Patients presenting these features began to be admitted in greater and greater numbers. Within a few months young adults began to appear in the outpatient department with parkinsonism. In some, a history of an acute illness could be ascertained. In others, the patients seemed to be ambulant from the beginning. Acute cases of the disease are now rarely seen, but chronic cases dating from as far back as I9I6 are still to be seen. Parkinsonism and loss of power of accommodation are the commonest features.

At first the disease was called botulism. Failure to discover the organism led to the name sleepy sickness. Economo called the disease encephalitis lethargica, thus combining its pathological and one of his clinical features.

\section{Aetiology.}

Encephalitis lethargica occurs sporadically and epidemically, chiefly in the cold seasons. No age is exempt, but it is most common during the first half of adult life. The virus is conveyed from healthy carriers by means of droplet infection. It gains access to the central nervous system by way of the peripheral neurons in the nasal passages. In I9I6, Economo transferred the disease to the monkey by intracerebral inoculation.

\section{Nature of Onset and Course.}

Usually, there is an acute onset with features indistinguishable from influenza. Pyrexia may last a week. As a rule there is marked drowsiness and diplopia. Sometimes, however, the patients are ambulant from the beginning, the movements of one or more limbs being slowed down. In these cases diplopia is not always present, and the disease is difficult to distinguish from paralysis agitans. But in encephalitis lethargica there is usually, in addition to parkinsonism, some loss of power of convergence. The majority of patients develop parkinsonian symptoms. The progress of an acute case can be reliably estimated by the respiration rate. A steady rise during the first four days of the illness is a very bad sign.

\section{Pathology.}

The brain is oedematous and congested. The reddened grey matter is prominent against the normal white matter.

Microscopically.-The vessels of the brain are congested. There is perivascular lymphocytic cuffing with absence of polymorpho-nuclear leucocytes. Demyelination does not occur.

Cerebrospinal fluid.- This is usually normal, but the lymphocytes and protein are occasionally raised in the acute state.

Parts of the nervous. system affected.-The lesion usually falls particularly on the basal 
ganglia, the cerebrum and the brain stem. The chief clinical signs arise from the lesion in the corpus striatum, dissociated automatic movements being prominent. The pyramidal system is rarely effected, thus, it is unusual to find extensor plantar responses in the disease. Objective sensory disturbances are also rare, and the deep reflexes are, as a rule, normal. Apart from disturbances of ocular movements the cranial nerves are rarely involved.

\section{Associated Automatic Control.}

The majority of cases, if not all of them, present both in the early and later phases of the disease some disturbance in associated automatic movement. The function of associated automatic control has been ascribed to the corpus striatum. Normally, when walking, the arms swing forwards and backwards, the right arm swinging forwards with the left leg, and the left arm swinging forwards with the right leg. Thus, the movements of one side of the body are automatically brought into relation with those of the opposite side. There are also associated automatic movements in the execution of movements of one limb, thus, when the fingers are extended there is an associated flexion of the wrist, and when the fingers are flexed in making a fist, there is an associated extension of the wrist. Moreover, there are associated automatic movements of the head and eyes. When looking up, down, or to the side, the eyes and head act together. A great number of associated automatic movements may be observed.

Neurons concerned in associated automatic control.-There are three of these, namely, (a) striato-rubral neurons, whose course is from the corpus striatum to the red nucleus. $(b)$ rubrospinal neurons whose course is from red nucleus to the anterior horn cells, and (c) lower motor neurons into which the impulses for associated automatic control pass to reach the muscles.

\section{The Relationship Between the Lesion and the Symptoms.}

The first relay of neurons, namely, the striato-rubral neurons are those effected in encephalitis lethargica, and it is due to a lesion in them that the principal clinical features occur. These are displayed as follows.

I. Delay in the execution of movements in response to the will. Normally, a familiar voluntary movement is executed more or less simultaneously with the will. In encephalitis lethargica there is a definite delay between the desire to make a movement and its execution. This delay may be either so slight as to be unrecognisable except by the patient, or so severe as to amount to a paralysis. This defect may occur in one or more limbs. Sometimes one arm alone is affected, and when the patient is instructed to extend the fingers it will be observed that there is no associated flexion of the wrist, or that the association is defective. Similarly, when a fist is made instead of the wrist extending, the joint is kept immobile. When both upper limbs are involved the movements appear to be waxlike. It is common to see such patients at mealtime holding a knife and fork, their arms remaining immobile above the table for long periods. Thus, an unusually long time is taken over meals. Indeed, it is sometimes necessary to feed the patients. But I have often observed patients who were unable to carry out useful acts, such as writing, eating, or dressing, catch a ball thrown to them and quickly return it. This is due to the integrity of the pyramidal system.

2. Changes in facial expression. Normally the facial expression is automatically responsive to the emotions which are, no doubt, constantly changing, and this change is concerned in the formation of the individual's countenance. In encephalitis lethargica there is a delay more or less marked in the reaction to an emotional experience. Instead of laughing in response to emotional stimulus there may be movements of the face, but they are expressionless and mask-like.

3. Disturbance of associated automatic control. (a) Movements of the head and eyes: in encephalitis lethargica instead of the head normally following the movements of the eyes, the head may remain more or less immobile; $(b)$ movements of the arms and legs: one or other or both arms may fail to swing when walking.

4. Spasm of divergence. Although ptosis, ophthalmoplegia and changes in the normal reaction and size of the pupils may occur in encephalitis lethargica, spasm of divergence is the commonest. The pupils usually react normally to light, but when the patient is instructed to follow the examiner's finger which approaches the patient's eye it will be found that the pupils do not contract, and that when the finger reaches close to the eyes there is a spasm of divergence 
seen in one or both eyes. The patient rarely complains of diplopia in the chronic state of the disease.

5. Tremor of the fingers.-The tremor presents a rhythmic, rolling character, but it is much finer, as a rule, than that seen in paralysis agitans.

6. Plasticity. The tone of the muscles in the limbs affected is more rigid than normal.

\section{Other Complications.}

Some of these are so rare as to be regarded almost in the nature of clinical curiosities. There are many, the chief of which are the following. Motor. Choreiform and athetoid movements occur and, very rarely, epileptic attacks. Sensory. Severe headache is very common. It is persistent and difficult to relieve. Respiratory. Hiccough, yawning, and spasmodic cough are all uncommon. Mental. Most patients retain their intellectual faculties, but behaviour disorders sometimes present themselves and dementia may occur. Sleep rhythm. A most troublesome feature, even in those not badly physically affected by the disease, is narcolepsy. The patients when working fall asleep and are unable to retain their employment. They may sleep badly during the night. Oculogyric crisis is characterised by a tonic conjugate deviation of the eyes upwards, or downwards. This may last for a few minutes or for hours. The frequency of the attacks varies. There may be long intervals between the attacks. The patients may pass from one attack into another for days, and then have a quiescent interval for months.

\section{Notification.}

All cases of encephalitis lethargica must be notified to the medical officer of health.

\section{Treatment.}

During the acute stage the treatment is that of an acute infection. The patients need not be isolated. No specific remedy is known. It is not possible to prevent either a second attack or the condition from developing into one of parkinsonism. For chronic cases stramoniumb and hyoscine are the favourite drugs. The former is given in increasing doses. Beginning with min. I5 of the tincture three times daily, the dose is gradually increased until mine 50 t.d.s. are reached. Hyoscine is perhaps the more beneficial. It is given as hyoscine hydrobromide gr. I/I50, with sodium bromide gr. Io; t.d.s.p.c.

\section{ENCEPHALITIS PERIAXIALIS}

\section{(Schilder's Disease)}

In I9I2, Paul Schilder published the clinical and post mortem findings of a case which he had mistakenly diagnosed as one of cerebral tumour. It was that of a girl of fourteen years, in whom he found right hemianopia. In the course of some twelve months she gradually developed right hemiplegia, aphasia, signs of increased intracranial tension, and, finally, incontinence. She died after seventeen months of illness. The autopsy showed diffuse sclerosis of both cerebral hemispheres.

\section{Aetiology.}

The disease occurs in the young, particularly in the very young, and affects both sexes equally. Although Schilder regarded it as inflammatory, its familial incidence suggests a primary degeneration.

\section{Pathology.}

The central white matter of the cerebral hemispheres is affected, either on one or on both sides, by a primary demyelination which ultimately leads to destruction of the axons. As a result a transluscent greyish or yellowish brown jelly-like substance is formed which, beginning in a small area of the central white matter, may extend throughout the whole of it. Neuroglial cells and neuroglial fibres are increased. The subcortical zone is the last to be involved, and the cortex remains normal. The direction of spread of the pathological process can only be inferred by the order of appearance of the clinical signs. As in Schilder's case, so in the majority of those seen, the early occurrence of homonymous hemianopia, without other clinical signs, 
suggests that the disease usually begins in the occipital white matter. Moreover, the early appearance of blindness indicates that the occipital lobes may be early affected on both sides. The lesion spreads forwards, and may extend as far as the prefrontal lobes on one or on both sides. One hemisphere may be affected before the other. Scattered patches of the disease may be found in the optic chiasma, cerebellar peduncles, and in the white matter of the brain stem.

\section{Symptoms.}

Cerebral function is gradually impaired until it is finally lost. The lesion in the occipital region. Destruction of the visual fibres on one side leads to contralateral homonymous hemianopia. Total blindness results from a lesion on both sides. There is neither optic atrophy nor alteration of the pupillary reflex to light because the neurons affected belong to the final relay of visual neurons which arise in the external geniculate body. In the very young great difficulties are sometimes experienced in determining the extent of loss of sight. The lesion in the temporal region causes deafness. The lesion in the post-Rolandic area gives rise to hemianaesthesia of a postural sense type. The lesion in the pre-Rolandic area causes hemiplegia with pyramidal signs. The lesion in the pre-frontal region causes amentia.

The cerebro-spinal fluid is usually normal, but there may be a slight increase, in cells and protein.

\section{Course and Prognosis.}

The disease is usually fatal within three years.

\section{Treatment.}

There is no known effective treatment.

\section{ACUTE DISSEMINATED ENCEPHALOMYELITIS}

(Devic's Disease)

In view of the lesion occurring in the optic nerve the disease is sometimes called neuromyelitis optica. It is characterised by diffuse myelitis with optic neuritis. ' Its cause is unknown. This disease was well described by C. Worster-Drought in this Journal (I936), 12, I98.

\section{Structures Involved.}

The spinal cord. Here the lesion may be confined to a few segments of the cord. On the other hand, many segments may be diseased. The pyramidal tracts alone or with other pathways may be involved. There is demyelination of the axis cylinders. Optic nerves. The fibres show demyelination.

\section{Signs of the Disease.}

As a result of the lesion in the spinal cord there is spastic paraplegia from the lesion in the pyramidal tracts. The deep reflexes are active, the plantars are extensor, and the abdominal reflexes are absent. When other tracts are diseased, corresponding signs accompany them. Sensory loss to pain and temperature is rare because their neurons are not myelinated.

The lesion in the optic nerves causes blindness.

\section{OTOGENIC ENCEPHALITIS}

In the course of otitis media with mastoiditis encephalitis without abscess formation sometimes occurs. It is characterised by headache, vomiting, and sometimes papilloedema. Localising signs, such as convulsions, aphasia, pyramidal, or cortico-sensory signs, may strongly suggest an abscess. But whereas cerebral abscess usually pursues an afrebile course, otogenic encephalitis is associated with an elevation of temperature. The cerebro-spinal fluid may be normal in each case. The condition is not easy to diagnose, but a definite plan should be followed when a discharging ear is accompanied by signs of increased intracranial tension.

Before the operation for mastoid. There should be no delay in performing a mastoid operation. 
After the operation for mastoid. (a) When the temperature is raised the dura should not be opened, nor the brain explored by a needle. If this is done the patient will rarely, if ever, survive. With the subsidence of temperature there may be a complete disappearance of the signs of increased intracranial tension with recovery. (b) When the temperature is not raised and there is strong indication that an abscess is present, it is desirable to exercise patience and not to hurry to open the brain. Great harm is often done by inserting a needle into different parts of brain in order to try to discover where is the pus. Small abscesses are very difficult to locate when operating. In my experience patients with abscess of the brain only recover when an incision is made straight into the abscess with the first cut.

-

\title{
ACUTE ASEPTIC MENINGITIS
}

\author{
By R. WYBURN-MASON, M.A., M.D.(Cantab), M.R.C.P.(Lond.) \\ (Registrar, National Hospital, Queen Square)
}

Occasionally in acute cases of meningitis no bacteria can be found in the C.S.F. either by microscopical examination or by culture. This condition has been termed "acute aseptic meningitis." Much difficulty has arisen owing to the confused nomenclature of conditions coming under this definition. The term has been applied to the following:-

(a) Meningitic reactions (pleocytosis, increased protein) secondary to spinal anaesthesia with novocaine and other drugs, following intrathecal lipiodol injection and intrathecal thera peutic injection of horse serum, which augments meningeal permeability and produces a pleo cytosis of perhaps roo cells per $\mathrm{cu}$. $\mathrm{mm}$. These reactions are not of great clinical importance and no further reference will be made to them.

(b) Hydrocephalus due to defective absorption of C.S.F. and not uncommonly seen as a late result of mastoiditis, otitis media, frontal sinusitis, etc. This is the so-called "otitic (or toxic) hydrocephalus" (Symonds I93I, I937). It is due to thrombosis of the superior longitudinal sinus by extension from the lateral or other venous sinuses. Papilloedema is conspicuous, but headache and vomiting slight. The C.S.F. is under increased pressure, but shows no other changes. This condition, strictly speaking, is not a form of meningitis and again no further reference to it will be made here. Those interested may consult the papers of Symonds.

(c) Meningitis associated with a cranial focus of infection, e.g. mastoiditis, and in which the C.S.F. is sterile. This has also been styled "sympathetic meningitis." The majority of cells in the fluid are polymorphonuclears.

(d) Meningitis in which there is a marked lymphocytosis in the C.S.F. and from some cases in which a virus has been isolated. The prognosis is good. This syndrome has been described by different authors under the following names:-acute aseptic meningitis: acute benign meningitis; acute idiopathic meningitis; epidemic meningitis serosa; benign aseptic purulent meningitis; and benign lymphocytic (chorio-) mengingitis.

The term "serous meningitis" possesses no meaning and should be discarded. It has been applied to all the above conditions in turn. The main distinction between the various forms of meningitis lies in the cellular reaction of the C.S.F. and on this the prognosis largely depends. It oo is simplest, therefore, to divide aseptic meningitis into acute aseptic purulent meningitis and acute aseptic lymphocytic (chorio-) meningitis, in which the cellular reactions of the meninges are mainly polymorphonuclear or mononuclear in type respectively.

\section{ACUTE ASEPTIC PURULENT MENINGITIS}

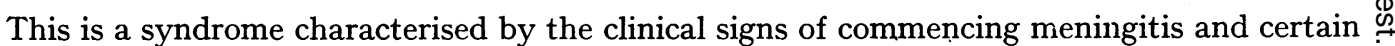
changes in the C.S.F. It is associated with otitis media, mastoiditis, extradural abscess, purulent sinusitis or brain abscess. Pathologically the condition is thought to be that of purulent 